\title{
Quaternionic Analytic Signal Using Atomic Functions
}

\author{
E. Ulises Moya-Sánchez and Eduardo Bayro-Corrochano \\ CINVESTAV, Unidad Guadalajara \\ Electrical and Computer Sciences Department \\ $\{$ emoya, edb\}@gdl. cinvestav.mx
}

\begin{abstract}
Atomic Functions are widely used in different applications in image processing, pattern recognition, computational physics and also in the digital interpretation of signal measurements. In 1D signals, is usual to compute the phase and the magnitude of a signal using the analytic signal (the signal and its Hilbert transform using complex numbers). However, for high dimensional signals the monogenic signal (the signal and its Riesz transform) has been used to obtain the local phase and orientation with good results. The main aim of this work is to present a new way to make the computation of the Hilbert transform using the atomic function. The computation of the Hilbert transform take relevance when the phase computation is required.
\end{abstract}

Keywords: Quaternion Algebra, Atomic functions, Image Processing, 2D Phase Information.

\section{Introduction}

The visual system is the most advanced of our senses. Therefore, it is easy to understand that the processing of the images plays an important role in human perception and computer vision implementation [89]. For $1 \mathrm{D}$ signals is usual to compute the phase and the magnitude using the signal and its Hilbert transform with a complex number [9]. Additionally, the phase information can be used to detect low-level geometric characteristics such as lines or edges 9517. Moreover, the phase can also be used to measure the local decomposition of the image according to its symmetries 9810 . As will be shown in this work, the atomic function $u p(x)$ in addition to hypercomplex numbers can be used as a building block to get multiple operations commonly used in image processing and analysis, such as low/high-pass filter, n-order derivatives, local phase, local amplitude, local orientation etc.

\section{Atomic Functions}

The Atomic Functions $A F$ are compactly supported infinitely differentiable solutions of differential equations with a shifted argument [1] i.e.

$$
L f(x)=\lambda \sum_{k=1}^{M} c(k) f(a x-b(k)),|a|>1,
$$


where $L=\frac{d^{n}}{d x^{n}}+a_{1} \frac{d^{n-1}}{d x^{n-1}}+\ldots+a_{n}$ is a linear differential operator with constant coefficients. In the $A F$ class, the function $u p(x)$ is the simplest and at the same time, the most useful primitive function to generate other kinds of atomic functions [1]. It satisfies the equation 1 as

$$
\begin{array}{r}
f(x)^{\prime}=2(f(2 x+1)-f(2 x-1)), \\
d u p(x)=2(u p(2 x+1)-u p(2 x-1))
\end{array}
$$

Function $u p(x)$ is infinitely differentiable but non-analytical; $u p(0)=1, u p(-x)=$ $u p(x)$. In general the Atomic Function up $(x)$ is generated by infinite convolutions of rectangular impulses. The function $u p(x)$ has the following representation in terms of the Fourier transform:

$$
u p(x)=\frac{1}{2 \pi} \int_{-\infty}^{\infty} e^{i u x} \prod_{k=1}^{\infty} \frac{\sin \left(u 2^{-k}\right)}{u 2^{-k}} d u .
$$

Figure 1 shows the $u p(x)$ and the Fourier Transform of $F(u p)$. Atomic windows were compared with classic ones [1] by means of the system of parameters such as: the equivalent noise bandwidth, the $50 \%$ overlapping region correlation, the parasitic modulation amplitude, the maximum conversion losses (in decibels), the maximum side lobe level (in decibels), the asymptotic decay rate of the side lobes (in decibels per octave), the window width at the six-decibel level, the coherent gain. All atomic windows exceed classic ones in terms of the asymptotic decay rate [1].
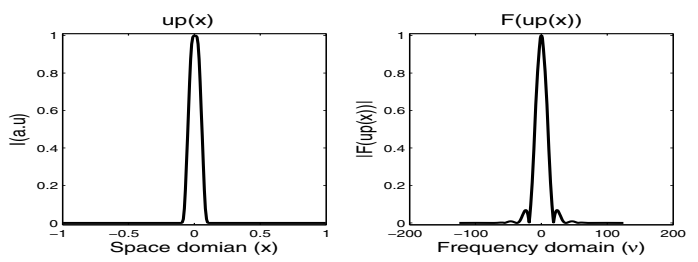

Fig. 1. Atomic function $u p(x)$ and the Fourier Transform of $u p(x)$

\section{Quaternion Algebra}

The even subalgebra $\mathcal{G}_{3,0,0}^{+}$(bivector basis) is isomorphic to $\mathcal{H}$, which is an associative, noncommutative, four-dimensional algebra that consists of one real element and three imaginary elements.

$$
q=a+b i+c j+d k a, b, c, d \in \Re
$$

The units $i, j$ obey the relations $i^{2}=j^{2}=-1, i j=k$. $\mathcal{H}$ is geometrically inspired, and the imaginary components can be described in terms of the basis 
of $\mathcal{R}^{3}$ space, $i \rightarrow e_{23}, j \rightarrow e_{12}, k \rightarrow e_{31}$. Another important property of $\mathcal{H}$ is the phase concept. A polar representation of $q$ is

$$
q=|q| e^{i \phi} e^{k \psi} e^{j \theta}
$$

where $|q|=\sqrt{q \bar{q}}$ where $\bar{q}$ is a conjugate of $q=a-b i-c j-d k$ and the angles $(\phi, \theta, \psi)$ represent the three quaternionic phases 3 .

\subsection{Quaternionic Atomic Function $Q u p(x, y)$}

Since any $2 \mathrm{D}$ function $f(x, y)$ can be split into even $(e)$ and odd $(o)$ parts [3],

$$
f(x, y)=f_{e e}(x, y)+f_{o e}(x, y)+f_{e o}(x, y)+f_{o o}(x, y)
$$

we can separate the four components of $u p(x, y)$ function and represent it as a quaternion as follows [1710]:

$$
\begin{aligned}
\operatorname{Qup}(x, y) & =u p(x, y)\left[\cos \left(w_{x}\right) \cos \left(w_{y}\right)+i\left(\sin \left(w_{x}\right) \cos \left(w_{y}\right)\right)+\right. \\
& \left.+j\left(\cos \left(w_{x}\right) \sin \left(w_{y}\right)\right)+k\left(\sin \left(w_{x}\right) \sin \left(w_{y}\right)\right)\right] \\
& =\text { Qup }_{e e}+i Q u p_{o e}+j Q u p_{e o}+k Q u p_{o o} .
\end{aligned}
$$

Figure 2 shows a $\operatorname{Qup}(x, y)$ in a space domain with its four components: the real part $Q u p_{e e}$ and the imaginary parts $Q u p_{e o}, Q u p_{o e}, a n d Q u p_{o o}$. We can see even and odd symmetries in the horizontal, vertical, and diagonal axes.
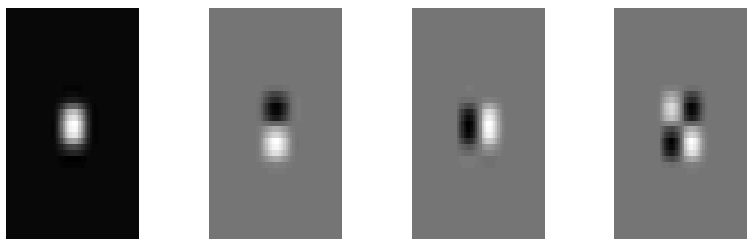

Fig. 2. From left to right. $Q u p_{e e}, Q u p_{o e}, Q u p_{e o}$, and $Q u p_{o o}$.

\section{Quaternionic Local Phase Information}

In this paper, we refer to the phase information as the local phase in order to separate the structure or geometric information and the amplitude in a certain part of the signal. Moreover, the phase information permits us to obtain invariant or equivariant 1 response. For instance, it has been shown that the phase has an invariant response to changes in image brightness and the phase can be used to measure the symmetry or asymmetry of objects [5198]. These invariant and

${ }^{1}$ Equivariance: monotonic dependency of value or parameter under some transformation. 
equivariant responses are the key part to link the low-level processing with the image analysis and the upper layers in computer vision applications.

The local phase means the computation of the phase at a certain position in a real signal. In $1 \mathrm{D}$ signals, the analytic signal based on the Hilbert transform $\left(f_{\mathbf{H}}(x)\right)[3]$ is given by

$$
\begin{array}{r}
f_{A}(f(x))=f(x)+i f_{\mathbf{H}}(x), \\
f_{A}(f(x))=|A| e^{i \theta},
\end{array}
$$

where $|A|=\sqrt{f(x)^{2}+f_{\mathbf{H}}(x)^{2}}$ and $\theta=\arctan \left(\frac{f(x)}{f_{\mathbf{H}}(x)}\right)$ permits us to extract the magnitude and phase independently. In $2 \mathrm{D}$ signals, the Hilbert transform is not enough to compute the magnitude and phase independently in any direction [18]. Then, in order to solve this, the quaternionic analytic (see Eq. 11) signal and the monogenic signal have been proposed by Bülow [3] and Felsberg [18, respectively. Until now, we have used an approximation of the quaternionic analytic signal based on the basis of $Q F T$ to extract some oriented axis symmetries.

Figure (3) contains, at the top, an image with lines and edges, at the center an image profile, and at the bottom the profiles of the three quaternionic phases. In phase profiles, we can distinguish between a line (even) and an edge (odd) using the phase $(\theta)$. These results are similar to the results reported by Granlund 9 using only a complex phase, because they used an image that changes in one direction.

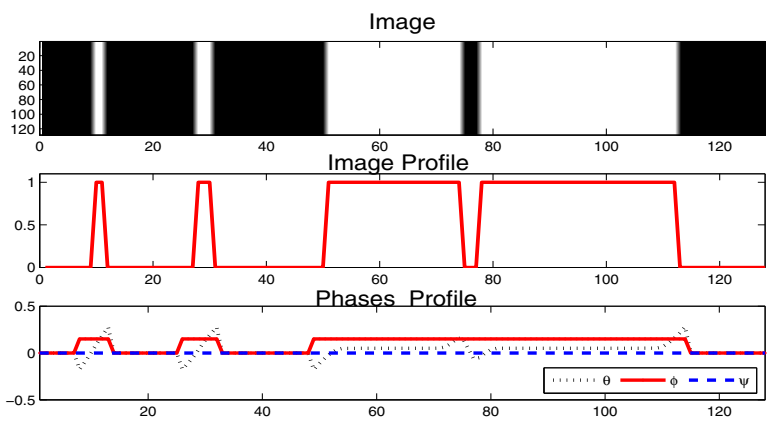

Fig. 3. From top to bottom: image, image profile, and three quaternionic phases: profile, line, and edge

\subsection{Quaternionic Analytic Signal}

The quaternionic analytic signal in the space domain is defined as 3 .

$$
f_{A}^{q}(x, y)=f(x, y)+i f_{\mathbf{H} i}(x, y)+j f_{\mathbf{H} j}(x, y)+k f_{\mathbf{H} k}(x, y),
$$

where $f_{\mathbf{H} i}(x, y)=f(x, y) * \frac{1}{\pi x}$ and $f_{\mathbf{H} j}(x, y)=f(x, y) * \frac{1}{\pi y}$ are the partial Hilbert transforms and $f_{\mathbf{H} k}(x, y)=f(x, y) * \frac{1}{\pi^{2} x y}$ is the total Hilbert transform. Bülow 
has shown that the $Q F T$ kernel is expressed in terms of the Hilbert transforms. The phases can be computed easily using a $3 \mathrm{D}$ rotation matrix $\mathcal{M}$, which can be factored into three rotations, $R=R_{x}(2 \phi), R_{z}(2 \psi), R_{y}(2 \theta)$, in the coordinate axes [3], i.e.,

$$
\begin{gathered}
\mathcal{M}(q)=\mathcal{M}\left(q_{1}\right) \mathcal{M}\left(q_{2}\right) \mathcal{M}\left(q_{3}\right) \\
q_{1}=e^{i \phi}, q_{2}=e^{j \theta}, q_{3}=e^{k \psi}, \\
\mathcal{M}(q)=\left(\begin{array}{ccc}
a^{2}+b^{2}-c^{2}-d^{2} & 2(b c-a d) & 2(b d+a c) \\
2(b c+a d) & a^{2}-b^{2}+c^{2}-d^{2} & 2(c d-a b) \\
2(b d-a c) & 2(c d+a b) & a^{2}-b^{2}-c^{2}+d^{2},
\end{array}\right)
\end{gathered}
$$

where $a, b, c$ and $d$ are defined in the Eq 5. Then, the phases are expressed by the following rules:

$$
\psi=-\frac{\arcsin (2(b c-a d))}{2}
$$

- If $\psi \in]-\frac{\pi}{4}, \frac{\pi}{4}\left[\right.$, then $\phi=\frac{\arg _{i}\left(\boldsymbol{q} \mathcal{T}_{j}(\overline{\boldsymbol{q}})\right)}{2}$ and $\theta=\frac{\arg _{j}\left(\mathcal{T}_{i}(\overline{\boldsymbol{q}}) \boldsymbol{q}\right)}{2}$.

- If $\psi= \pm \frac{\pi}{4}$, then select either $\phi=0$ and $\theta=\frac{\arg _{j}\left(\mathcal{T}_{k}(\overline{\boldsymbol{q}}) \boldsymbol{q}\right)}{2}$ or $\theta=0$ and $\phi=$ $\frac{\arg _{i}\left(\boldsymbol{q} \mathcal{T}_{k}(\overline{\boldsymbol{q}})\right)}{2}$.

- If $e^{i \phi} e^{k \psi} e^{j \theta}=-\boldsymbol{q}$ and $\phi \geq 0$, then $\phi \rightarrow \phi-\pi$.

- If $e^{i \phi} e^{k \psi} e^{j \theta}=-\boldsymbol{q}$ and $\phi<0$, then $\phi \rightarrow \phi+\pi$.

The phase ranges are $(\phi, \theta, \psi) \in\left[-\pi, \pi\left[\times\left[-\frac{\pi}{2}, \frac{\pi}{2}\left[\times, \pi\left[-\times\left[-\frac{\pi}{4}, \frac{\pi}{4}\right]\right.\right.\right.\right.\right.$.

\subsection{Hilbert Transform Using $\boldsymbol{A F}$}

The main aim of this section is to present a new way to make the computation of the Hilbert transform using the atomic function. The Hilbert transform and the derivative are closely related, and the Hilbert transform can actually be done using a derivative and some convolution $(\star)$ properties [14]:

$$
\begin{aligned}
f \star(g \star h) & =(f \star g) \star h \\
\nabla(f \star g) & =\nabla f \star g=f \star \nabla g,
\end{aligned}
$$

where $f(x, y), g(x, y) h(x, y) \in \mathcal{R}^{2}, \nabla=e_{1} \frac{\partial}{\partial x}+e_{2} \frac{\partial}{\partial y}$. If $g(x, y)=-\frac{1}{\pi} \log (|x|)$ $\log (|y|)$ where $\log (|x|)$ is the fundamental solution of Laplace equation. Moreover if we use the convolution distribution properties, we can express the Hilbert transform and the partial Hilbert transform (see Eq. 11) as

$$
f_{\mathbf{H}_{\mathbf{i}}}(x, y)=\frac{\partial f(x, y)}{\partial x} \star-\frac{1}{\pi} \log (|x|)
$$




$$
\begin{aligned}
f_{\mathbf{H}_{\mathbf{j}}}(x, y) & =\frac{\partial f(x, y)}{\partial y} \star-\frac{1}{\pi} \log (|y|) \\
f_{\mathbf{H}_{\mathbf{k}}}(x, y) & =\frac{\partial^{2} f(x, y)}{\partial x \partial y} \star-\frac{1}{\pi^{2}} \log (|x|) \log (|y|)
\end{aligned}
$$

and we can use the convolution association property to get the equation of a certain part of the signal in terms of $\operatorname{dup}(x)$ (see Eq 3):

$$
\begin{aligned}
f_{\mathbf{H}_{\mathbf{i}}}(x, y) & =f(x, y) \star\left(\operatorname{dup}(x, y)_{x} \star-\frac{1}{\pi} \log (|x|)\right) \\
f_{\mathbf{H}_{\mathbf{j}}}(x, y) & =f(x, y) \star\left(\operatorname{dup}(x, y)_{y} \star-\frac{1}{\pi} \log (|y|)\right) \\
f_{\mathbf{H}_{\mathbf{k}}}(x, y) & =f(x, y) \star\left(\operatorname{dup}(x, y)_{x y} \star-\frac{1}{\pi^{2}} \log (|x|) \log (|y|) .\right)
\end{aligned}
$$

The figure 4 shows the $u p(x)$ and its Hilbert transform.
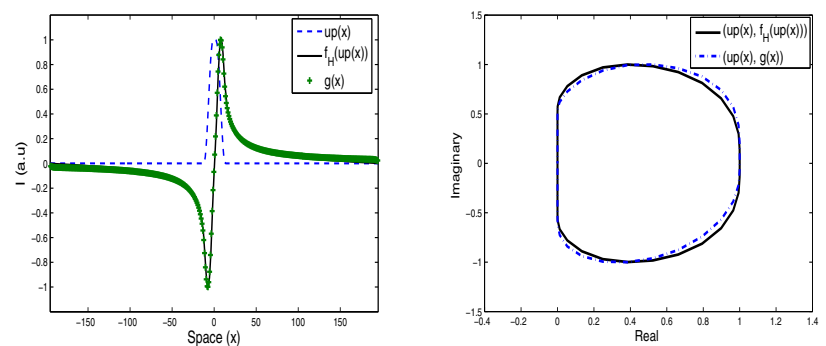

Fig. 4. $u p(x)$, its Hilbert transform and Eq21 Rigth Dot-line $u p(x)$ (real part) and the Hilbert transform (imaginary).

\section{Results}

Figure 5illustrates the convolution of the first derivative, $\operatorname{dup}(x, y)$, and a shadow chessboard. The convolution with dup can be used as an oriented change detector with a simple rotation, the convoluted images has a low response in the shadow part.

Figure (6) illustrates an image with lines and geometric objects. In this case, we can use the quaternionic phases to extract different oriented edges of geometric objects or different oriented line textures. The $\theta$ and $\phi$ phases detect lines or edges in some vertical and horizontal directions, while the $\psi$ phase detects the diagonal response and the corners in squares or geometric figures. Even if the geometric objects have lines inside, different illuminations or positions, the edges of each square can be detected in $\phi$ and $\theta$ images. Moreover, in the $\phi$ phase, the horizontal lines are highlighted, whereas the vertical lines dont appear. The $\theta$ phases show a similar result, but in this case the vertical edges and lines are highlighted. In the $\psi$ phase, the vertical lines or edges are highlighted. 

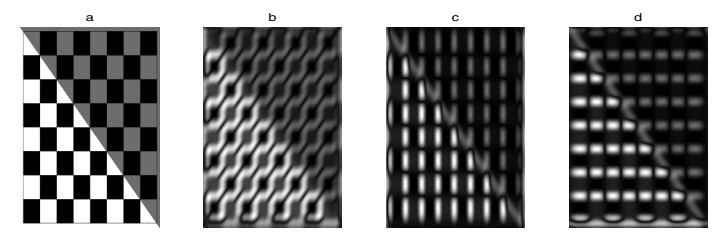

Fig. 5. Convolution of $d u p(x, y)$ with the test image. (a) Test image; (b)result of the convolution of the image with $\operatorname{dup}\left(x, y, 0^{\circ}\right)$; (c) $\operatorname{dup}\left(x, y, 45^{\circ}\right)$; (d) $d u p\left(x, y, 135^{\circ}\right)$.
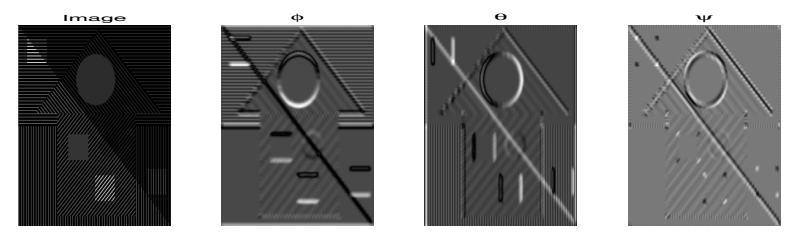

Fig. 6. Image and $2 \mathrm{D}$ quaternionic phases. A texture based on lines can be detected or discriminated, and at the same time, the phase information can highlight the edges.

\section{Conclusion}

We have shown that the $\operatorname{Qup}(x, y)$ is useful to detect lines or edges in a specific orientation using the quaternionic phase concept. Additionally, an oriented texture can be chosen using the quaternionic phases. As an initial step, we have shown how to do the image analysis of geometric objects in $\Re^{2}$ using the symmetry. As in other applications in geometric algebra $G A$, we can take advantage of the constraints. Since the information from the three phases is independent of illumination changes, algorithms using the quaternionic atomic function can be less sensitive than other methods based on the illumination changes. These results motivated us to find other invariants such as rotation invariants using the Riesz transform. In a future work, we can develop a complete computer vision approach based on $G A$.

\section{References}

1. Kravchenko, V.F., Perez-Meana, H.M., Ponomaryov, V.I.: Adaptive digital processing of multidimensional signals with applications. In: MOSCOW FIZMATLIT (2009)

2. Bayro-Corrochano: Geometric Computing for Wavelet Transforms, Robot Vision, Learning, Control and Action. Springer, London (2010)

3. Bülow, T.: Hypercomplex Spectral Signal Representations for the Processing and Analysis of Images. Christian- Albert, Kiel University. Ph.D Thesis (1999)

4. Jähne, B.: Digital Image Processing. Springer, Germany (2002)

5. Kovesi, P.: Invariant measures of images features from phase information. University of Western Australia. PhD Thesis (1996) 
6. Bayro, E.: Geometric Computing for Wavelet Transforms, Robot Vision, Learning, Control and Action. Springer, London (2010)

7. Hestenes, D.: New Foundations for Classical Mechanics, 2nd edn. Kluwer Academic Publishers, Arizona (2002)

8. Josef Bigun.: Vision with Direction. Springer (2006)

9. Granlund, G., Kutsson, H.: Signal Processing for Computer Vision. Lippincott Williams and Wilkins, Philaphia (2002)

10. Moya-Sánchez, E.U., Vázquez-Santacruz, E.: A Geometric Bio-inspired Model for Recognition of Low-Level Structures. In: Honkela, T. (ed.) ICANN 2011, Part II. LNCS, vol. 6792, pp. 429-436. Springer, Heidelberg (2011)

11. Dorts, L., Mann, S.: Geometric algebra: A computational framework for geometrical applications. In: IEEE Computer Graphics ans Applications, pp. 25-31 (2002)

12. Kovesi, P.: Invariant measures of images features from phase information. $\mathrm{PhD}$ thesis, University of Western Australia, Autralia (1996)

13. Lennart, W.: Local feature detection by higher order riesz transforms on images. Master thesis, Christian- Albert, Kiel University, Kiel Germany (2008)

14. Svensson, B.: A Multidimensional Filtering Framework with Applications to Local Structure Analysis and Image Enhancement. PhD thesis, Linkoping University, Linkoping, Sweden (2008)

15. Kolodyazhnya, V.M., Rvachev, V.A.: Cybernetics and Systems Analysis, vol. 43 (2007)

16. Treil, S., Petermichl, S., Volberg, A.: Why the riesz transforms are averages of the dyadic shifts? Publ. Mat. 46(6) (2002)

17. Moya-Sánchez, E.U., Bayro-Corrochano, E.: Quaternion Atomic Function Wavelet for Applications in Image Processing. In: Bloch, I., Cesar Jr., R.M. (eds.) CIARP 2010. LNCS, vol. 6419, pp. 346-353. Springer, Heidelberg (2010)

18. Felsberg, M.: Low-level image processing with the structure multivector. PhD thesis, Christian- Albert, Kiel University, Kiel, Germany (2002) 